\title{
Von Willebrand Disease: What does the future hold?
}

2

3 Cécile V. Denis ${ }^{1}$, Sophie Susen ${ }^{2}$, and Peter J. Lenting ${ }^{1}$,

$4{ }^{1}$ Laboratory for Hemostasis, Inflammation \& Thrombosis, Unité Mixed de Recherche S1176,

5 Institut National de la Santé et de la Recherche Médicale, Université Paris-Saclay, 94276 Le

6 Kremlin-Bicêtre, France

$7 \quad{ }^{2} \mathrm{CHU}$ Lille, Université de Lille, Institut National de la Santé et de la Recherche Médicale Unité

8 1011, Institut Pasteur de Lille, Lille, France; CHU Lille, Hematology and Transfusion, Lille,

9 France

10

Correspondence: $\quad$ Peter J. Lenting, INSERM U1176, 80 rue du General Leclerc, 94276 Le KremlinBicêtre, France Tel: +33149595651; Fax: +33146719472;

Short title: Von Willebrand disease: What does the future hold?

17

Article type: Perspective

19

Abstract: 242 words

Main text (without figure legends): 4000 words

22 Figures: 2

23 References: 86 


\section{Abstract}

2 Von Willebrand disease (VWD) is characterized by its heterogeneous clinical manifestation,

3 which complicates its diagnosis and management. The clinical management of VWD has

4 remained essentially unchanged over the last 30 years or so, using von Willebrand factor (VWF)

5 concentrates, desmopressin and anti-fibrinolytic agents as main tools to control bleeding. This

6 is in contrast to hemophilia A, for which a continuous innovative path has led to novel

7 treatment modalities.

8 Despite current VWD management being considered effective, quality-of-life studies

9 consistently reveal a higher than anticipated burden of VWD on patients, which is particularly

10 true for women. Apparently, despite our perceived notion of current therapeutic efficiency,

11 there is space for innovation with the goal to reach superior efficacy. Developing innovative

12 treatments for VWD is complex, especially given the heterogeneity of the disease and the

13 multifunctional nature of VWF. In this perspective article, we describe several potential 14 strategies that could provide the basis for future VWD treatments. These include genetic 15 approaches such as gene therapy using dual-vector adeno-associated virus and transcriptional 16 silencing of mutant alleles. Furthermore, protein-based approaches to increase FVIII levels in 17 VWD-type 3 or $2 \mathrm{~N}$ patients will be discussed. Finally, antibody-based options to interfere with 18 VWF degradation (for congenital VWD-type 2A or acquired Von Willebrand syndrome-type 2A) 19 or increase endogenous VWF levels (for VWD-type 1) will be presented. By highlighting these 20 potential strategies, we hope to initiate an innovative path, which ultimately would allow us 21 to better serve VWD patients and their specific needs. 


\section{Introduction}

Von Willebrand disease (VWD) is an inherited bleeding disorder resulting from quantitative or qualitative deficiencies in von Willebrand factor (VWF), a plasma multimeric glycoprotein controlling platelet adhesion and aggregation. ${ }^{1}$ Although VWD is expected to affect men and women equally, there are more women diagnosed with VWD due to gynecological-related hemostatic challenges. The real prevalence of VWD has been a matter of debate for many years, ranging from as high as $1.3 \%$ when taking into account epidemiological studies ${ }^{2}$ to 100 cases per million when considering patients with clinically relevant bleeding symptoms. ${ }^{3}$ VWD is traditionally classified in three types. VWD-type 1 is characterized by a partial quantitative deficiency in VWF. VWD-type 2 refers to qualitative VWF defects with a number of subtypes (2A, 2B, $2 \mathrm{~N}$ and $2 \mathrm{M}$ ) according to the patients' functional defect. Finally, VWDtype 3 , also called severe VWD, is manifested by a virtually complete deficiency in VWF. ${ }^{1}$

Bleeding symptoms are usually considered less serious than hemophilia-related symptoms. Although that may be true for mild VWD patients, specific comparative studies revealed that the bleeding-profile between VWD-type 3 and severe hemophilia $A$ is more similar than expected..$^{4,5}$ Nevertheless, prophylactic treatment remains relatively rare in VWD patients.

To develop alternative treatments for managing VWD in the $21^{\text {st }}$ century, it will be crucial to understand the challenges faced by VWD patients and to take into account the disease specificities.

\section{Challenges in the diagnosis of VWD}

Given the heterogeneity of the disease that is observed sometimes within the same family or even in the same patient throughout his/her lifetime, VWD has been and remains to this day, a complex entity to diagnose ${ }^{6}$ and to manage, partially explaining the difference between prevalence and actually diagnosed patients. Laboratory testing of VWD outside highly specialized centers is a challenge in itself, and the link between mild phenotypes and a VWF defect is not always straightforward. In addition, bleeding history relies on a subjective interpretation by the patients, their parents as well as the clinician. This is particularly true with regard to menstrual bleeding, a subject not easily discussed both inside and outside the family circle. Drs. Weyand and James recently noted that while $76 \%$ of men with VWD are diagnosed by 10 years, $50 \%$ of the women are not diagnosed by 12 years of age. ${ }^{7}$ Although 
1 this could partially be explained by a first bleeding episode at different ages, it has also been shown that the diagnostic delay following the first bleeding episode is longer in women than men. ${ }^{8}$ Delayed diagnosis may result in missed treatment opportunities and increased morbidity (both medical and psychological). It is thus important to increase knowledge and awareness of VWD among (general) clinicians and public.

\section{VWD and quality of life}

What objective tools do we actually rely on to define that VWD is a mild bleeding disorder in most cases? First and primary is the clinical management of patients, since many VWD patients are followed in specialized hemophilia treatment centers. The health care professionals managing patients are highly-trained hematologists who have the necessary expertise to evaluate the disease burden. However and as stated maybe provocatively by Dr. Kouides "VWD on clinical grounds is primarily a disease of females". ${ }^{9}$ We are now increasingly learning how gender bias may have unwillingly affected patient's care in many areas. ${ }^{10-12}$ Whether such an issue has contributed to underestimate VWD severity in terms of physical and psychological consequences is unclear. Yet, there seems to be a parallel between the increasing number of female hematologists and the greater recognition of significant VWDassociated morbidities, especially when related to women reproductive health. ${ }^{13-15}$ The second tool to evaluate disease severity are health-related quality of life (HR-QoL) studies, widely accepted as crucial instruments of patient-centered care. ${ }^{16}$ For VWD, a few studies have addressed this issue using either 1) validated HR-QoL questionnaires, 2) questionnaires not officially validated or 3 ) through interviews. All studies have reported significantly decreased quality of life in VWD patients. The largest study (509 patients) was performed in the Netherlands using the generic Short Form (SF)-36 and reported decreased vitality (score measuring energy and fatigue) in both men and women with VWD. ${ }^{17}$ Smaller studies performed in Canada (102 patients, SF-36 questionnaire), Finland (47 patients, Medical Outcomes Study-36 questionnaire), Sweden (30 female patients, SF-36 questionnaire), Canada again (28 patients, Health Utility Index Mark-2 and Mark-3) or United Kingdom (57 female patients, general questionnaire) not only confirmed a lower quality of life for VWD patients compared to the general population, but for studies in which both men and women were included, most reported a difference between both genders. ${ }^{18-22}$ Studies focusing primarily on women clearly indicate how the burden of VWD is borne disproportionately by 
1 women. Heavy menstrual bleeding and its associated pain (observed in $32-100 \%$ of patients), ${ }^{23}$ post-partum hemorrhage (in 15-60\% of patients), ${ }^{13}$ and hysterectomy (performed in $10-26 \%$ of patients), ${ }^{24}$ are common in VWD women. ${ }^{15,25}$ Qualitative interview-based studies about HRQoL or lived experiences are even more striking with women reporting a considerable impairment in their lives. ${ }^{26-28}$ Obviously not all VWD patients are affected by severe bleeding symptoms, a fact that can mitigate some of the HR-QoL studies where only a modest impact was reported, such as in the large Dutch study. Focusing on subgroups can probably give better ideas of the situation. A perfect example is illustrated in another Dutch study focusing on VWD patients with joint bleeds. ${ }^{29}$ The authors show that $23 \%$ of patients from the Dutch VWD cohort self-reported joint bleeds. Not only is this percentage higher than anticipated, but joint bleeds were reported in VWD-type 1 as well as in VWD-type 2 or VWD-type 3, even if factor VIII (FVIII) levels exceeded 10\%. Joint bleeds were associated with increased consumption of factor concentrates, joint pain and lower HR-QoL, suggesting a "significant burden of arthropathy in VWD". ${ }^{29}$ Another factor that can impact the results of the HR-QoL studies is the absence of diseasespecific instruments. ${ }^{23}$ All the validated HR-QoL questionnaires used so far are generic instruments. A VWD-specific QoL has been developed, ${ }^{30}$ but although it was mentioned that the questionnaire was validated in a German study (Wil-QoL) and a French study (WISH-QoL), no publications are yet available.

Altogether, these different studies illustrate the higher than anticipated burden of VWD on patients and suggest that VWD classification in types 1,2 and 3, although crucial to understand the physiopathology of the disease is not always a good proxy for clinical severity. The actual patient's phenotype is not only driven by the VWD phenotype, but also by burden in terms of hospitalizations, surgical interventions, drug consumption, gynecological visits, sick leaves and other objective parameters. This burden is only in part included in the bleeding score used to characterize the disease (Bleeding Assessment Tool of the International Society on Thrombosis and Hemostasis). However, this tool is cumulative and more adapted to diagnosis rather than reevaluation of the bleeding tendency. To better understand the evolution of the patient's phenotype and associated burden, an alternative score could be designed to determine spontaneous symptomatology in the presence and absence of treatment. Undoubtedly, despite our perceived notion of therapeutic efficacy of current VWD treatments, 
1 improved management of patients is necessary to address patient's needs in an optimal

2 fashion.

4 Current treatment

5 Over the last 30 years, many reviews related to the clinical management of VWD have been published (see eg. ${ }^{1,24,31-38}$ ) and guidelines were recently published. ${ }^{39}$ Without extensively revisiting the topic, some relevant issues can be highlighted. The most relevant message is that the toolkit for the treatment of VWD has remained essentially unchanged over these 30 years. Already in 1991, Dr. Aledort described the use of intermediate-purity factor concentrates and desmopressin as main approaches for the management of VWD, as well as adjunctive anti-fibrinolytic and estrogenic therapy. ${ }^{31}$ It then took until 2005 for the development of a purer plasma-derived VWF concentrate, poor in FVIII activity..$^{40}$ As for the first recombinant VWF concentrate, it is only since 2015 that it became available in the USA and 2017 in Europe. ${ }^{41}$

When establishing a parallel with progresses made in the clinical management of hemophilia A over the same period of time, the contrast is particularly striking (Figure 1). While a parallel development was observed in terms of intermediate-purity concentrates (which contain both VWF and FVIII) and desmopressin in the 1980s, a real divergence started in the 1990s with the development of monoclonal antibody-purified and recombinant FVIII concentrates. At that time, who would have guessed that it would take another 20 years for comparable products to be available for VWD? But research did not stand still in the hemophilia field and while VWD management was only trying to fill the gap, nothing short of a revolution was looming on the other side of the VWF/FVIII partnership. Indeed, during the last decade, hemophilia A treatment has soared with several novel therapeutic strategies being available or in late clinical development. ${ }^{42-45}$ In particular, gene therapy is reaching maturity and therefore for the first time in history, a real cure for the disease is within sight.

Thus, innovation in VWD treatment has basically stopped and the limitations of current treatments have not been addressed. Of course, the arrival of a recombinant VWF concentrate is an important step forward. However, without neglecting its specific merits, the availability of this recombinant VWF concentrate is not changing the essentials of VWD management, and prophylactic treatment will still require multiple intravenous infusions per week. The invasive nature of intravenous administration is associated with an increased risk 
1 of infections, it is inconvenient, and it is challenging in the very young and old to find adequate 2 venous access for repeated injections. Furthermore, prophylactic treatment using concentrates is expensive and not affordable world-wide. Finally, a major hindrance to the use of prophylaxis is that the criteria for introduction of prophylaxis for VWD patients (particularly women with heavy menstrual bleeding) are not as well defined as for patients with hemophilia. As a consequence, the benefit or need for prophylaxis are less well recognized. ${ }^{46}$

The advantages of desmopressin are numerous, and validate its status as the "treatment of choice" in VWD-type 1 patients. It can be administered in different ways (IV, subcutaneous or intranasal), is relatively cheap, and without risk of transmittable disease. It will transiently achieve adequate post-treatment levels of VWF and FVIII in $80 \%$ of cases. ${ }^{47,48}$ However, there are also some limitations. It has variable effectiveness in type $2 A \& 2 M$, and is usually considered for minor bleeding/surgery. ${ }^{47}$ Moreover, this medication is contra-indicated for VWD-type 2B, while its application in VWD-type 3 is ineffective since no FVIII or VWF is released from the endothelial storage-organelles. ${ }^{47}$ Importantly, desmopressin is primarily a antidiuretic, with VWF release being an off-target effect. A number of side-effects concomitant to desmopressin use have also been reported (transient headaches, facial flushing, hypotension, hyponatremia and mild tachycardia), which are generally mild and welltolerated. ${ }^{49,50}$ Nevertheless, a recent VWD coping survey presented by Kalot et al. revealed that patients/caregivers display more concern about the side effect profile of desmopressin compared to health care professionals, and seem to prefer a prophylactic approach. ${ }^{51}$ Furthermore, the repetitive use of desmopressin may result in reduced responsiveness (tachyphylaxis) due to exhaustion of the VWF storage-organelles. ${ }^{52}$ Finally, it is relevant to mention that desmopressin is a monopolized product by Ferring Pharmaceuticals, which could compromise availability in case of production issues (see eg. https://www.ferringusa.com/press/ferring-us-issues-voluntary-nationwide-recall-of-ddavpnasal-spray-10-mcg-0-1ml-desmopressin-acetate-nasal-spray-10-mcg-0-1ml-stimate-nasalspray-1-5-mg-ml-due-to-superpotency/).

Hormonal treatment is commonly used to reduce heavy menstrual bleeding in VWD. However, as for desmopressin, increased VWF/FVIII levels are a mere secondary effect of hormonal treatment, which in itself has its own side effects and contra-indications. These are not always 
minor, as for example the use of progesterone has been associated with an increased risk of spheno-orbital osteomeningiomas.

In conclusion, YES there are some efficient treatments for VWD, but NO this does not mean that we should stop trying to improve these treatments with the goals of reaching superior efficacy, improving quality of life of the patients and finally curing the disease.

\section{The challenge of innovation in VWD management}

What can be done to improve VWD treatment? Unsurprisingly, there is not a simple answer to that question. Increasing prophylactic treatment would be a first step, as the introduction of prophylaxis for VWD patients appears to significantly reduce the rate of bleeding episodes and the need for hospitalization. ${ }^{53,54}$ Nevertheless, prophylactic treatment did not fully prevent against the occurrence of bleeding episodes. ${ }^{54}$ In addition, the current concentrates have similar issues as the FVIII concentrates that spurred the development of novel treatment strategies for hemophilia, including a relatively short half-life requiring multiple intravenous infusions per week. Designing VWF variants with a prolonged half-life could be an option to reduce infusion frequency. Such a strategy would not be such a major challenge to implement but besides an abstract published in 2006 which showed its validity, no follow-up development has been put in place. ${ }^{55}$ However, to be truly innovative, we as a scientific community need to think of alternative ways that are adapted to the patients' situation, rather than being limited to VWF concentrates or desmopressin. It is with such novel mindset that we will be able to break the glass ceiling and come up with the VWD-specific equivalents of fitusiran or emicizumab. In order to move in this direction, we first need to identify the patients who will better benefit from a more personalized approach, a concept increasingly recognized as relevant. ${ }^{37,56}$ As part of this identification process, we should realize that severe VWD is not necessarily restricted to VWD-type 3. Patients with VWD-type 1 or 2 may sometimes experience bleeding phenotypes similar in severity than some VWD-type 3 patients. ${ }^{57}$ Knowing the VWD subtype of the patients will be crucial to adapt treatment but should not represent a limit in our perception and appreciation of disease severity. There are patients who experience major bleeds and some who have frequent minor bleeds and our challenge is to address the different needs of the bleeders. 
1 In the following sections, we will describe a number of potential approaches that could change

2 the current paradigm of how VWD is treated or can be treated in the upcoming years. Of note,

3 we will not address specifically the platelet dysfunction described in some patients with VWD-

4 type $2 \mathrm{~B}^{58}$ since targeting the VWF gene defect would also resolve the platelet phenotype.

\section{Novel strategies: genetic approaches} Gene therapy

To really cure VWD, gene therapy represents the ultimate goal, especially as it seems to be successful for both hemophilia A and B. However, the challenges to express VWF via gene therapy appear quite daunting. A first obstacle relates to its multimerization. Several mouse studies have shown that functional VWF can be efficiently expressed in hepatocytes of VWFdeficient mice, either transiently ${ }^{59-62}$ or for long-term expression. ${ }^{63,64}$ However, hepatocytes are not well-equipped to produce sufficiently multimerized VWF, and over time the hemostatically active VWF multimers seem to disappear. ${ }^{63}$ So targeting the endothelium will be necessary for efficient protein processing.

Size represents a second hurdle: the VWF CDNA is too large to be incorporated in adenoassociated viruses (AAV), the type of vectors most used in clinical trials. Successful incorporation into lentiviruses has been reported, but data on efficient in vivo expression is yet lacking. ${ }^{65,66}$

In an attempt to overcome both obstacles, our team in collaboration with Federico Mingozzi's team has designed a dual-AAV vector approach, in which the cDNA of VWF is divided over two AAV-particles, which in turn are adapted to specifically target endothelial cells. We show that such an approach is technically feasible although the current expression levels are too low to be clinically meaningful. ${ }^{67}$

But even if we overcome these obstacles, there is another hurdle that we cannot ignore: if VWF is finally expressed at reasonable levels in the endothelial cells of the patient, how can we avoid the incorporation of dominant-negative mutants into the VWF multimer, when being expressed in the same cells as the transgene VWF? Indeed, Bowman et al. reported that the inheritance pattern for VWD-type 3 proved co-dominant in approximately $50 \%$ of the families included in their study. ${ }^{68}$

Perhaps expression of full-length VWF is not always necessary. In VWD-type 2N, and also some VWD-type 3 patients, the bleeding complications are associated with the low levels of FVIII, 
and increasing FVIII levels would help to reduce bleeding in joints and soft tissues. For those patients, a gene therapy approach using the $N$-terminal portion of VWF (D1-D2-D'-D3 domains) would result in normalization of FVIII levels, as the presence of the dimeric D'D3-fragment protects FVIII from degradation and premature clearance ${ }^{69}$ Advantage of this approach is that multimerization is not a requirement, the cDNA (about $3.8 \mathrm{~kb}$ ) easily fits into the AAV-vector and the protein can be expressed in hepatocytes. Preliminary studies in mice showed that such an approach is viable from a technical point of view. ${ }^{69}$

\section{Transcriptional silencing/gene-editing}

10 Rather than trying to express functional VWF, another approach would be to silence expression of mutant VWF. This makes even more sense given the dominant-negative character of many of the VWF mutations. A first example of this approach was reported by Casari et al., who demonstrated that siRNA-mediated targeting of the mRNA breakpoint of an in-frame deletion mutant improved protein expression and multimerization. ${ }^{70}$ Recently, we applied this siRNA-approach to a mouse model of VWD and confirmed the restoration of VWF multimers in plasma by silencing the dominant-negative allele. ${ }^{71}$ To find a broader application for this technology, de Jong et al. designed siRNAs targeting common single-nucleotide polymorphisms in the VWF mutant allele, while leaving expression of the wild-type allele unaffected. ${ }^{72}$

Alternatively, another option could be to correct the VWF gene itself, using gene-editing approaches such as Crispr-Cas9. Little information on this approach in view of VWD currently exists, with the exception of a study investigating the bi-allelic deletion of the VWF gene in endothelial colony forming cells (ECFCs, also known as blood outgrowth endothelial cells). ${ }^{73}$ Both siRNA and gene-editing approaches are attractive, but one of the technical difficulties includes the specific targeting of sufficient numbers of endothelial cells. Currently, we do not know how many endothelial cells would need to be targeted to achieve sufficient production of functional VWF multimers. Additional research on these promising strategies is clearly needed at this point.

\section{Cellular therapy}

Ex vivo cellular therapy represents another method to introduce cells expressing functional 
1 ECFCs were transduced using lentiviral vectors containing the human VWF cDNA. One day

2 after transduction, cells indeed expressed functional multimerized VWF at levels 3-fold higher compared to normal ECFCs. However, expression levels to decreased 7-fold over a 4-week period during the expansion phase, which could be due to in vitro culturing of these cells. While a similar approach could also be applied using endothelial cells derived from induced pluripotent stem cells, human somatic cell therapy approaches are currently limited by the ability to generate sufficient amounts of cells to achieve physiological relevant expression levels after transplantation, the uncertain life-span of transplanted cells and the occurrence of genetic changes in the cells. ${ }^{74}$ Progress on cellular therapies in other disease will certainly be helpful for the future development of this approach regarding VWD.

\section{Novel strategies: protein-based strategies}

Compensating FVIII-deficiency in VWD

Some of the bleeding complications in VWD-type $2 \mathrm{~N}$ and type 3 patients are related to low FVIII levels, and improving FVIII levels could thus be beneficial. Infusing standard recombinant FVIII is not a viable option, since FVIII will be extremely-short lived in the absence of protection conferred by VWF. On the other hand, using FVIII/VWF concentrates in type $2 \mathrm{~N}$ patients could lead to an excess of VWF, potentially posing a thrombotic risk. ${ }^{75,76}$ An alternative solution may soon become available with the development of BIVV001, a novel FVIII-fusion variant which circulates independently of endogenous VWF, having a half-life 3-4 times longer than recombinant FVIII. ${ }^{77,78}$ Although being developed for hemophilia A, this molecule could provide a novel treatment option for VWD-type $2 \mathrm{~N}$ patients to correct FVIII-deficiency without increasing VWF levels.

Another approach was described by Weyand and colleagues, related to a VWD-type 3 patient who had developed allo-antibodies against both FVIII and VWF and experienced recurrent bleeding episodes. ${ }^{79}$ To compensate for FVIII-deficiency in this patient, treatment with emicizumab was initiated. Emicizumab is a bispecific antibody that mimics to some extent the cofactor function of FVIII. ${ }^{43,80}$ Emicizumab prophylaxis resulted in improved bleed prevention and reduced treatment burden. ${ }^{79}$

Increasing endogenous VWF levels 
1 Desmopressin is often the first choice of treatment in VWD-type 1, as it will temporarily normalize endogenous levels of the otherwise functional VWF molecule. Due to the limitations associated with desmopressin detailed earlier, it would be of interest to develop novel strategies to increase endogenous VWF levels in a more sustainable approach. Various options could be designed in this respect. For instance, after the discovery that interleukin-11 infusion was associated with an increase in VWF levels in both mouse and canine models, its clinical benefit was evaluated in two Phase II studies. ${ }^{81-84}$ Although some clinical benefit following subcutaneous application was observed, the increase in VWF levels was modest (1.1-1.5 fold)..$^{83,84}$ No additional clinical studies seem to be registered at Clinicaltrials.gov. Another strategy includes the use of single-domain antibodies (nanobodies) fused to an albumin-binding moiety (eg. peptide or nanobody), for instance, a VWF-binding nanobody that is fused to an albumin-binding peptide. ${ }^{85}$ In this approach, such fusion protein would bridge circulating VWF to albumin, taking advantage of the long albumin half-life, and induce a prolonged half-life for VWF and consequently, increase VWF levels. Preliminary mouse studies revealed that pre-incubating VWF with a VWF-binding nanobody fused to an albuminbinding peptide (KB-VWF-013-ABP) resulted in a 7-fold increased VWF half-life. Moreover, subcutaneous application of this fusion protein was associated with a 4-8 fold increase in VWF- and FVIII-levels over a period of 7 days. ${ }^{85}$ This approach thus holds great promises but needs to be further confirmed in different preclinical models.

\section{Preventing VWF degradation}

Excessive degradation of VWF is a particular problem in congenital VWD-type 2A(IIA), but also in some variants of acquired von Willebrand syndrome (aVWs), for instance in those having severe aortic stenosis or patients requiring mechanical circulatory support. While congenital VWD-type $2 A$ could be treated using VWF concentrates, this is trickier in aVWs since it seems conceivable that the infused VWF will also be susceptible to degradation while passing through the pumps. To reduce VWF degradation, antibodies can be used to interfere with ADAMTS13-VWF interactions. Importantly, inhibition of VWF degradation should be limited to avoid thrombotic side effects as seen in thrombotic thrombocytopenic purpura. A few years ago, we described an anti-VWF antibody (Mab508) targeting the ADAMTS13-docking site at the VWF-D4 domain. ${ }^{86}$ This antibody interferes with excessive VWF degradation, while leaving basal degradation intact. Indeed, the presence of Mab508 led to the conservation of high 
1 molecular weight-multimers in ex vivo whole-blood perfusions systems. ${ }^{86}$ Given the

2 advantages of antibody-based treatment over concentrate-based treatments (eg. long half-

3 life, eventual subcutaneous administration), such antibodies could be valuable assets in managing patients characterized by excessive VWF degradation.

\section{Forward-looking perspective}

The heterogeneous nature of VWD implies that a "one size fits all" approach will never have the potential to address all the patients' needs. However, the management of VWD patients currently follows such a trend. As highlighted in the last few paragraphs, research has not stood still and various solutions have been or are under investigation, although more could definitely be done in this regard. The main roadblock, in our view, appears at the next level, the translation of research results in potential industrial partnerships, allowing development of future treatments. So what are the biggest obstacles? Market size is certainly a major hurdle with few patients likely to benefit from each of the approaches highlighted previously. Another difficulty comes from the difference in perception of disease burden between health care professionals and the patients themselves. Maybe it is time to stop comparing bleeding in hemophilia and bleeding in VWD. All HR-QoL studies tell us that VWD patients have a significantly impaired quality of life. Patient's advocacy has therefore a critical role to play in order to bring all stakeholders around the same table in order to prepare the future. No patient should be left behind. Our main aspiration is that ten years from now, if we were to write a review about VWD management, such a review will be quite different than the ones published for the last 30 years.

\section{Acknowledgements:}

Financial support by the Agence Nationale de la Recherche (ANR-17-RHUS-0011) is gratefully acknowledged. This perspective could not have been written without the input of Dr. Olivier Christophe and Dr. Caterina Casari.

\section{Author contributions:}

All authors contribution to the conception, writing and editing of this manuscript.

\section{Disclosures:}


1 CVD, SS and PJL are inventors on patents owned by Inserm related to bleeding disorders. CVD,

2 SS and PJL are co-founders of Laelaps Therapeutics.

3

4 Legends

Figure 2: Potential avenues to enrich the arsenal of treatment options for VWD

Examples of potential novel genetic- or protein-based strategies for the management of VWD are highlighted. Some approaches could be used to serve all VWD patients, whereas others will be type-specific. Abbreviations used in the figure: AAV: adeno-associated virus; ECs: endothelial cells; ECFCs: endothelial colony forming cells (also referred to as blood outgrowth endothelial cells); ECMO: extra-corporal membrane oxygenation; FVIII: factor VIII; IL-11: interleukin-11; LVAD: left ventricular assist device; TALENs: transcription activator-like effector nucleases; VWD: von Willebrand disease; VWs: von Willebrand syndrome. Portions of this figure were created using Biorender.com.

A schematic overview of when novel treatment options became available for hemophilia $A$ and VWD. High-purity and recombinant concentrates (orange) arrived 20 years later for VWD compared to hemophilia A. Extended half-life variants, non-factor therapies and gene therapy (red) have found their way to the clinic or are in late-stage clinical development for hemophilia A, whereas they are not yet at the horizon with regard to VWD. 
1. Leebeek FW, Eikenboom JC. Von Willebrand's Disease. $N$ Engl J Med. 2016;375(21):2067-2080.

2. Nichols WL, Hultin MB, James AH, et al. von Willebrand disease (VWD): evidence-based

3. Sadler JE, Mannucci PM, Berntorp E, et al. Impact, diagnosis and treatment of von Willebrand disease. Thromb Haemost. 2000;84(2):160-174.

4. Lak M, Peyvandi F, Mannucci PM. Clinical manifestations and complications of

5. Silwer J. von Willebrand's disease in Sweden. Acta Paediatr Scand Suppl. 1973;238:1-

8. Atiq F, Saes JL, Punt MC, et al. Major differences in clinical presentation, diagnosis and

6. James PD, Connell NT, Ameer B, et al. ASH ISTH NHF WFH 2021 guidelines on the diagnosis of von Willebrand disease. Blood Adv. 2021;published online 12 January 2021. DOI 10.1182/bloodadvances.2020003265.

7. Weyand AC, James PD. Sexism in the management of bleeding disorders. Res Pract Thromb Haemost. 2020;5(1):51-54. management of men and women with autosomal inherited bleeding disorders. EClinicalMedicine. 2021; doi.org/10.1016/j.eclinm.2021.100726.

9. Kouides PA. Females with von Willebrand disease: 72 years as the silent majority. Haemophilia. 1998;4(4):665-676.

10. Greenwood BN, Carnahan S, Huang L. Patient-physician gender concordance and increased mortality among female heart attack patients. Proc Natl Acad Sci U S A. 2018;115(34):8569-8574.

11. Regitz-Zagrosek V. Sex and gender differences in health. Science \& Society Series on Sex and Science. EMBO Rep. 2012;13(7):596-603.

12. Roter DL, Hall JA, Aoki Y. Physician gender effects in medical communication: a metaanalytic review. JAMA. 2002;288(6):756-764. 
1 13. Castaman G, James PD. Pregnancy and delivery in women with von Willebrand disease. Eur J Haematol. 2019;103(2):73-79.

14. Laffan M, Sathar J, Johnsen JM. von Willebrand disease: Diagnosis and treatment, treatment of women, and genomic approach to diagnosis. Haemophilia. 2020;doi: 10.1111/hae.14050.

15. Lavin M, Aguila S, Dalton N, et al. Significant gynecological bleeding in women with low von Willebrand factor levels. Blood Adv. 2018;2(14):1784-1791.

16. Snyder CF, Jensen RE, Segal JB, Wu AW. Patient-reported outcomes (PROs): putting the patient perspective in patient-centered outcomes research. Med Care. 2013;51(8 Suppl 3):S73-79.

17. de Wee EM, Mauser-Bunschoten EP, Van Der Bom JG, et al. Health-related quality of life among adult patients with moderate and severe von Willebrand disease. J Thromb Haemost. 2010;8(7):1492-1499.

18. Barr RD, Sek J, Horsman J, et al. Health status and health-related quality of life associated with von Willebrand disease. Am J Hematol. 2003;73(2):108-114.

19. Govorov I, Ekelund L, Chaireti R, et al. Heavy menstrual bleeding and health-associated quality of life in women with von Willebrand's disease. Exp Ther Med. 2016;11(5):1923-1929.

20. Kadir RA, Sabin CA, Pollard D, Lee CA, Economides DL. Quality of life during menstruation in patients with inherited bleeding disorders. Haemophilia. 1998;4(6):836-841.

21. Solovieva S. Clinical severity of disease, functional disability and health-related quality of life. Three-year follow-up study of 150 Finnish patients with coagulation disorders. Haemophilia. 2001;7(1):53-63.

22. Xu Y, Deforest M, Grabell J, Hopman W, James P. Relative contributions of bleeding scores and iron status on health-related quality of life in von Willebrand disease: a cross-sectional study. Haemophilia. 2017;23(1):115-121.

23. Von Mackensen S. Quality of life in women with bleeding disorders. Haemophilia. 2011;17 Suppl 1:33-37.

24. Kujovich JL. von Willebrand's disease and menorrhagia: prevalence, diagnosis, and management. Am J Hematol. 2005;79(3):220-228. 
1 25. Kouides PA, Phatak PD, Burkart P, et al. Gynaecological and obstetrical morbidity in women with type I von Willebrand disease: results of a patient survey. Haemophilia. 2000;6(6):643-648.

26. Kirtava A, Drews C, Lally C, Dilley A, Evatt B. Medical, reproductive and psychosocial experiences of women diagnosed with von Willebrand's disease receiving care in haemophilia treatment centres: a case-control study. Haemophilia. 2003;9(3):292-297.

27. Marshall AL, Dasari H, Warner ND, Grill DE, Nichols WL, Pruthi RK. Self-reported reproductive health experiences in women with von Willebrand disease: a qualitative interview-based study. J Obstet Gynaecol. 2019;39(2):288-290.

28. Arya S, Wilton P, Page D, et al. "Everyhing Was Blood When it Comes to Me": Understanding the Lived Experiences of Women with Inherited Bleeding Disorders. J Thromb Haemost. 2020;18(12):3211-3221.

29. van Galen KPM, Meijer K, Vogely HC, et al. Joint surgery in von Willebrand disease: a multicentre cross-sectional study. Haemophilia. 2016;22(2):256-262.

30. Von Mackensen S, Federici A. Quality of life assessment in patients with von Willebrand's disease: development of a first disease-specific instrument (VWD-QOL). Poster presentation at the annual congress of the Society of Thrombosis and Haemostasis Research, Dresden, 21-25 February 2007, P126. 2007.

31. Aledort LM. Treatment of von Willebrand's disease. Mayo Clin Proc. 1991;66(8):841846.

32. Kruse-Jarres R, Johnsen JM. How I treat type 2B von Willebrand disease. Blood. 2018;131(12):1292-1300.

33. Lavin M, O'Donnell JS. New treatment approaches to von Willebrand disease. Hematology Am Soc Hematol Educ Program. 2016;2016(1):683-689.

34. Lavin M, O'Donnell JS. How I treat low von Willebrand factor levels. Blood. 2019;133(8):795-804.

35. Leebeek FWG, Atiq F. How I manage severe von Willebrand disease. Br J Haematol. 2019;187(4):418-430.

36. Mannucci PM. How I treat patients with von Willebrand disease. Blood. 2001;97(7):1915-1919.

37. Phua CW, Berntorp E. A personalized approach to the management of VWD. Transfus Apher Sci. 2019;58(5):590-595. 
1 38. Tosetto A, Castaman G. How I treat type 2 variant forms of von Willebrand disease. Blood. 2015;125(6):907-914.

39. Connell NT, Flood V, Brignardello-Petersen R, et al. ASH ISTH NHF WFH 2021 guidelines on the management of von Willebrand disease. Blood Adv. 2021;published online 12 January 2021. DOI 10.1182/bloodadvances.2020003264.

40. Goudemand J, Scharrer I, Berntorp E, et al. Pharmacokinetic studies on Wilfactin, a von Willebrand factor concentrate with a low factor VIII content treated with three virusinactivation/removal methods. J Thromb Haemost. 2005;3(10):2219-2227.

41. Mannucci PM, Kempton C, Millar C, et al. Pharmacokinetics and safety of a novel recombinant human von Willebrand factor manufactured with a plasma-free method: a prospective clinical trial. Blood. 2013;122(5):648-657.

42. Chowdary P. Anti-tissue factor pathway inhibitor (TFPI) therapy: a novel approach to the treatment of haemophilia. Int J Hematol. 2020;111(1):42-50.

43. Kitazawa T, Igawa $T$, Sampei $Z$, et al. A bispecific antibody to factors IXa and X restores factor VIII hemostatic activity in a hemophilia A model. Nat Med. 2012;18(10):15701574.

44. Polderdijk SG, Adams TE, Ivanciu L, Camire RM, Baglin TP, Huntington JA. Design and characterization of an APC-specific serpin for the treatment of hemophilia. Blood. 2017;129(1):105-113.

45. Sehgal A, Barros S, Ivanciu L, et al. An RNAi therapeutic targeting antithrombin to rebalance the coagulation system and promote hemostasis in hemophilia. Nat Med. 2015;21(5):492-497.

46. Miesbach W, Berntorp E. Translating the success of prophylaxis in haemophilia to von Willebrand disease. Thromb Res. 2021;199:67-74.

47. Federici $A B$. The use of desmopressin in von Willebrand disease: the experience of the first 30 years (1977-2007). Haemophilia. 2008;14 Suppl 1:5-14.

48. Mannucci PM. New therapies for von Willebrand disease. Blood Adv. 2019;3(21):34813487.

49. Miesbach W, Krekeler S, Duck O, et al. Clinical assessment of efficacy and safety of DDAVP. Hamostaseologie. 2010;30 Suppl 1:S172-175.

50. Stoof SC, Cnossen MH, de Maat MP, Leebeek FW, Kruip MJ. Side effects of desmopressin in patients with bleeding disorders. Haemophilia. 2016;22(1):39-45. 
1 51. Kalot MA, Al-Khatib M, Connell NT, et al. An international survey to inform priorities for new guidelines on von Willebrand disease. Haemophilia. 2020;26(1):106-116.

52. Mannucci PM, Bettega D, Cattaneo M. Patterns of development of tachyphylaxis in patients with haemophilia and von Willebrand disease after repeated doses of desmopressin (DDAVP). Br J Haematol. 1992;82(1):87-93.

53. Holm E, Carlsson KS, Lovdahl S, Lail AE, Abshire TC, Berntorp E. Bleeding-related hospitalization in patients with von Willebrand disease and the impact of prophylaxis: Results from national registers in Sweden compared with normal controls and participants in the von Willebrand Disease Prophylaxis Network. Haemophilia. 2018;24(4):628-633.

54. Peyvandi F, Castaman G, Gresele P, et al. A phase III study comparing secondary longterm prophylaxis versus on-demand treatment with vWF/FVIII concentrates in severe inherited von Willebrand disease. Blood Transfus. 2019;17(5):391-398.

55. Turecek PL, Scheiflinger F, Siekmann J, et al. Biochemical and Functional Characterization of PEGylated rVWF. Blood. 2006;108 (11): 1021.

56. Hazendonk H, Heijdra JM, de Jager NCB, et al. Analysis of current perioperative management with Haemate $((R)) P /$ Humate $P((R))$ in von Willebrand disease: Identifying the need for personalized treatment. Haemophilia. 2018;24(3):460-470.

57. Federici $A B$, Bucciarelli $P$, Castaman $G$, et al. The bleeding score predicts clinical outcomes and replacement therapy in adults with von Willebrand disease. Blood. 2014;123(26):4037-4044.

58. Casari $C$, Berrou $E$, Lebret $M$, et al. von Willebrand factor mutation promotes thrombocytopathy by inhibiting integrin alphallbbeta3. $J$ Clin Invest. 2013;123(12):5071-5081.

59. De Meyer SF, Vandeputte N, Pareyn I, et al. Restoration of plasma von Willebrand factor deficiency is sufficient to correct thrombus formation after gene therapy for severe von Willebrand disease. Arterioscler Thromb Vasc Biol. 2008;28(9):1621-1626.

60. Golder M, Pruss $\mathrm{CM}$, Hegadorn $\mathrm{C}$, et al. Mutation-specific hemostatic variability in mice expressing common type 2B von Willebrand disease substitutions. Blood. 2010;115(23):4862-4869. 
61. Marx I, Christophe OD, Lenting PJ, et al. Altered thrombus formation in von Willebrand factor-deficient mice expressing von Willebrand factor variants with defective binding to collagen or GPIIbllla. Blood. 2008;112(3):603-609.

62. Rayes J, Hollestelle MJ, Legendre $P$, et al. Mutation and ADAMTS13-dependent modulation of disease severity in a mouse model for von Willebrand disease type 2B. Blood. 2010;115(23):4870-4877.

63. Portier I, Vanhoorelbeke K, Verhenne S, et al. High and long-term von Willebrand factor expression after Sleeping Beauty transposon-mediated gene therapy in a mouse model of severe von Willebrand disease. J Thromb Haemost. 2018;16(3):592-604.

64. Quiviger M, Giannakopoulos A, Verhenne S, et al. Improved molecular platform for the gene therapy of rare diseases by liver protein secretion. Eur J Med Genet. 2018;61(11):723-728.

65. De Meyer SF, Vanhoorelbeke K, Chuah MK, et al. Phenotypic correction of von Willebrand disease type 3 blood-derived endothelial cells with lentiviral vectors expressing von Willebrand factor. Blood. 2006;107(12):4728-4736.

66. Wang $\mathrm{L}$, Rosenberg JB, De BP, et al. In vivo gene transfer strategies to achieve partial correction of von Willebrand disease. Hum Gene Ther. 2012;23(6):576-588.

67. Barbon E, Kawecki C, Marmier S, et al. Development of a dual hybrid AAV vector for endothelial-targeted expression of von Willebrand factor. Gene Ther. 2021.

68. Bowman $M$, Tuttle A, Notley $C$, et al. The genetics of Canadian type 3 von Willebrand disease: further evidence for co-dominant inheritance of mutant alleles. J Thromb Haemost. 2013;11(3):512-520.

69. Yee A, Gildersleeve RD, Gu S, et al. A von Willebrand factor fragment containing the D'D3 domains is sufficient to stabilize coagulation factor VIII in mice. Blood. 2014;124(3):445-452.

70. Casari C, Pinotti M, Lancellotti S, et al. The dominant-negative von Willebrand factor gene deletion p.P1127_C1948delinsR: molecular mechanism and modulation. Blood. 2010;116(24):5371-5376.

71. Campioni $\mathrm{M}$, Legendre $\mathrm{P}$, Loubiere $\mathrm{C}$, et al. In vivo modulation of a dominant-negative variant in mouse models of von Willebrand disease type 2A. J Thromb Haemost. 2021;19(1):139-146. 
72. de Jong A, Dirven RJ, Boender J, et al. Ex vivo Improvement of a von Willebrand Disease Type 2A Phenotype Using an Allele-Specific Small-Interfering RNA. Thromb Haemost. 2020;120(11):1569-1579.

73. Schillemans $\mathrm{M}$, Kat $\mathrm{M}$, Westeneng J, et al. Alternative trafficking of Weibel-Palade body proteins in CRISPR/Cas9-engineered von Willebrand factor-deficient blood outgrowth endothelial cells. Res Pract Thromb Haemost. 2019;3(4):718-732.

74. Halliwell J, Barbaric I, Andrews PW. Acquired genetic changes in human pluripotent stem cells: origins and consequences. Nat Rev Mol Cell Biol. 2020;21(12):715-728.

75. Franchini M. Thrombotic complications in von Willebrand disease. Hematology. 2006;11(1):49-52 .

76. Rietveld IM, Lijfering WM, le Cessie S, et al. High levels of coagulation factors and venous thrombosis risk: strongest association for factor VIII and von Willebrand factor. J Thromb Haemost. 2019;17(1):99-109.

77. Konkle BA, Shapiro AD, Quon DV, et al. BIVV001 Fusion Protein as Factor VIII Replacement Therapy for Hemophilia A. N Engl J Med. 2020;383(11):1018-1027.

78. Seth Chhabra E, Liu T, Kulman J, et al. BIVV001, a new class of factor VIII replacement for hemophilia $A$ that is independent of von Willebrand factor in primates and mice. Blood. 2020;135(17):1484-1496.

79. Weyand AC, Flood VH, Shavit JA, Pipe SW. Efficacy of emicizumab in a pediatric patient with type 3 von Willebrand disease and alloantibodies. Blood Adv. 2019;3(18):27482750.

80. Lenting PJ, Denis CV, Christophe OD. Emicizumab, a bispecific antibody recognizing coagulation factors IX and X: how does it actually compare to factor VIII? Blood. 2017;130(23):2463-2468.

81. Denis CV, Kwack K, Saffaripour S, et al. Interleukin 11 significantly increases plasma von Willebrand factor and factor VIII in wild type and von Willebrand disease mouse models. Blood. 2001;97(2):465-472.

82. Olsen EH, McCain AS, Merricks EP, et al. Comparative response of plasma VWF in dogs to up-regulation of VWF mRNA by interleukin-11 versus Weibel-Palade body release by desmopressin (DDAVP). Blood. 2003;102(2):436-441.

83. Ragni MV, Jankowitz RC, Jaworski K, Merricks EP, Kloos MT, Nichols TC. Phase II prospective open-label trial of recombinant interleukin-11 in women with mild von 

645.

3

4

84. Ragni MV, Novelli EM, Murshed A, Merricks EP, Kloos MT, Nichols TC. Phase II prospective open-label trial of recombinant interleukin-11 in desmopressinunresponsive von Willebrand disease and mild or moderate haemophilia A. Thromb Haemost. 2013;109(2):248-254.

85. Kawecki C, Aymé G, Casari C, et al. Development of nanobodies fused to albumin binding peptide: a tool to increase plasmatic levels of endogenous proteins. Res Pract Thromb Haemost. 2017;1 (Suppl 1):OC-38.4.

86. Rauch A, Legendre P, Christophe OD, et al. Antibody-based prevention of von Willebrand factor degradation mediated by circulatory assist devices. Thromb Haemost. 2014;112(5):1014-1023. 
Figure 1: Evolution of treatment modalities for hemophilia A and von Willebrand disease

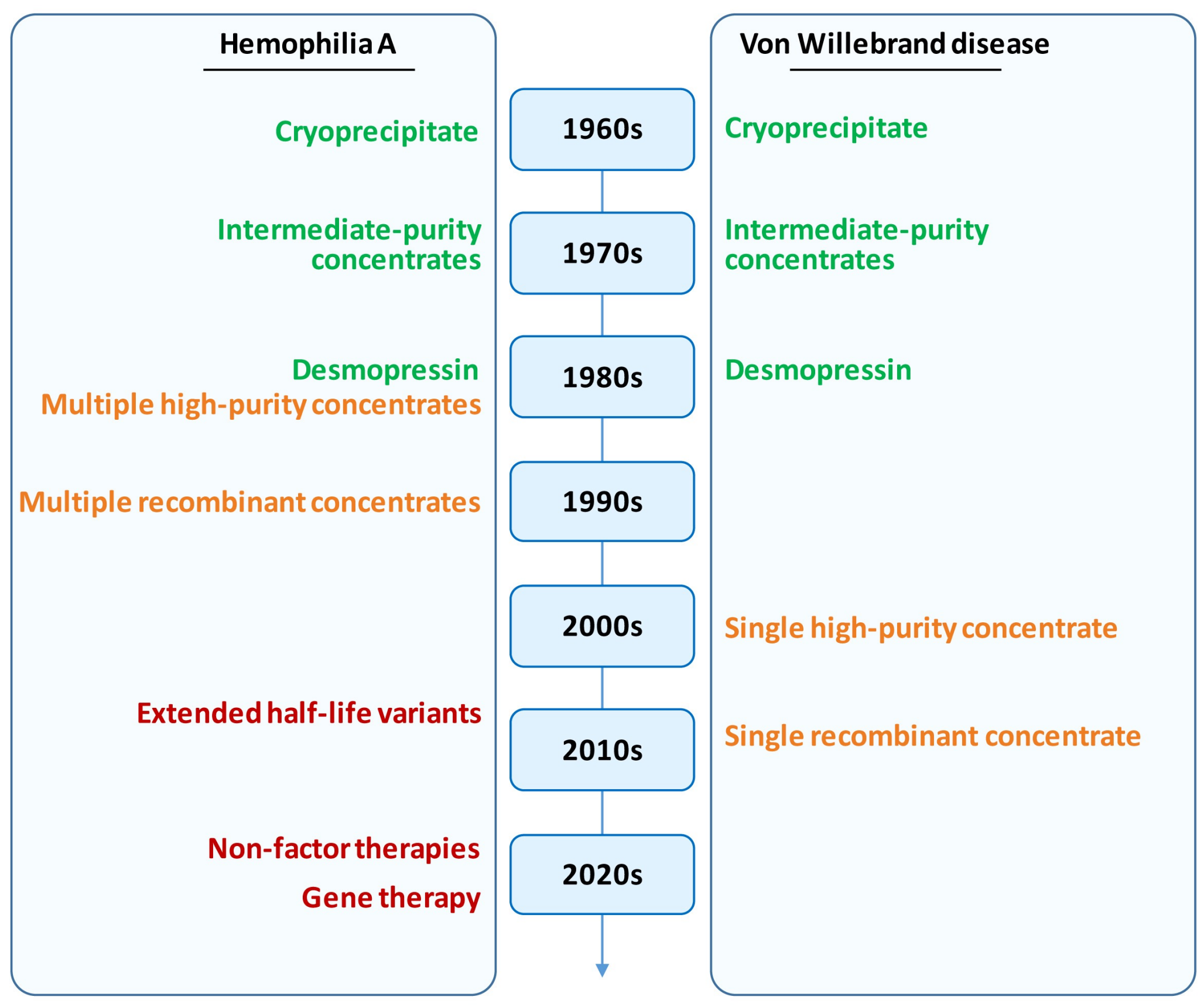


Figure 2: Potential avenues to enrich the arsenal of treatment options for VWD

\section{Genetic approaches}

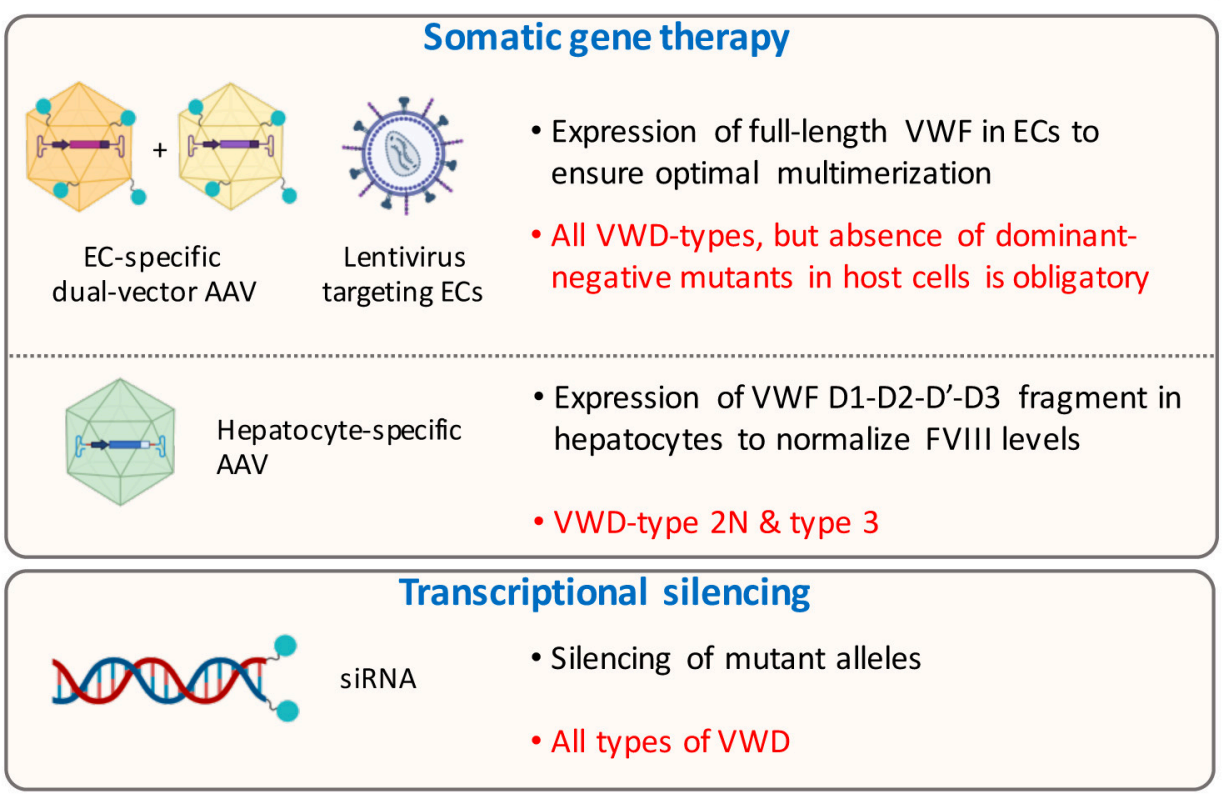

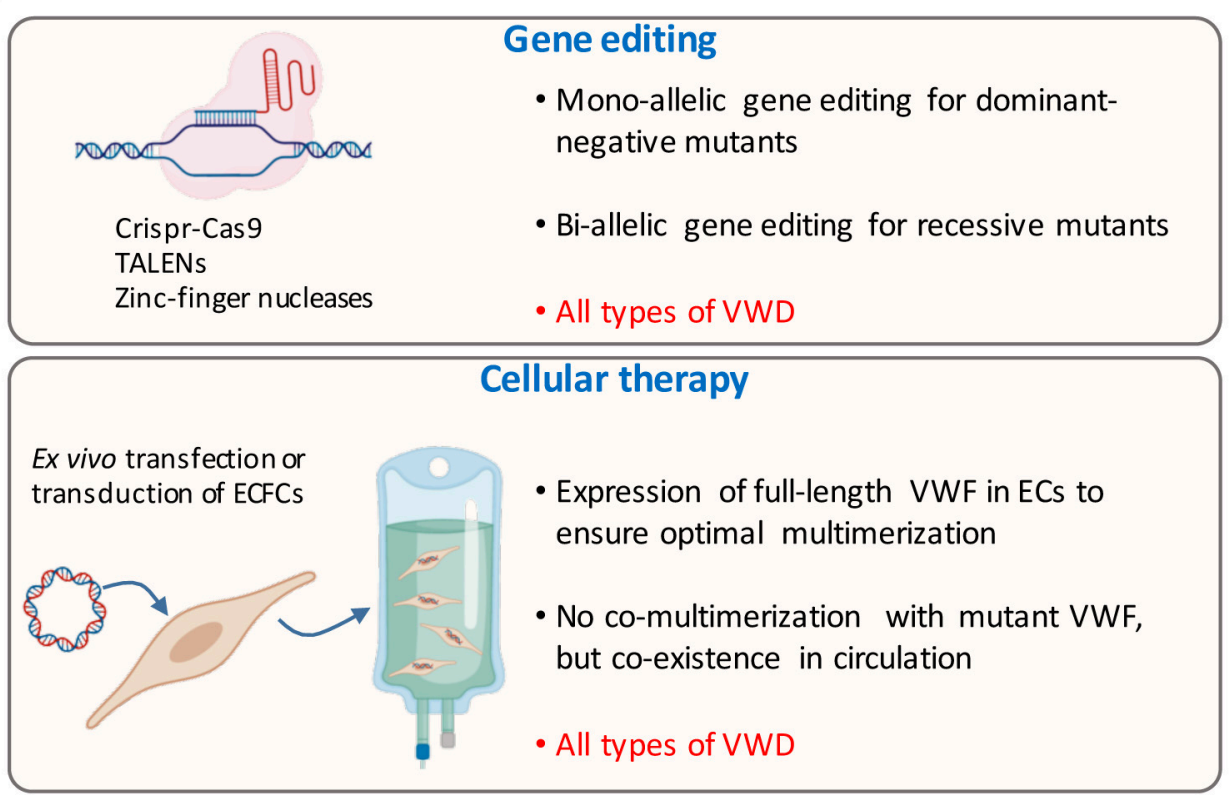

\section{Protein-based therapies}

Compensating FVIII deficiency
- BIVV001 is a FVIII-variant that circulates
independently of endogenous VWF
- Emicizumab is a bispecific antibody mimicking
FVIII-cofactor function \& can be given
subcutaneously
- VWD-type $2 \mathrm{~N}$ and type 3

Increasing endogenous VWF levels
- IL-11 stimulates synthesis of VWF, resulting in a
modest rise of VWF levels (1.1-1.5-fold)
- Bispecific nanobodies may delay clearance of
endogenous $\mathrm{VWF} \&$ can be given subcutaneously
- VWD-type 1 and type $2 \mathrm{M}$

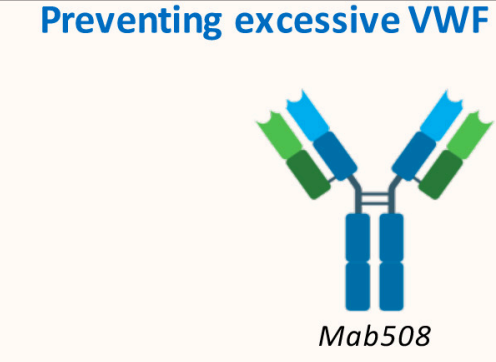

- Mab508 impairs VWF-ADAMTS13 interactions, thereby conserving high-molecular weight multimers

- VWD-type 2A (IIA)

- Acquired VWs-type 2A (including severe aortic stenosis, LVAD, ECMO,...) 